\title{
Zero distribution of polynomials satisfying a differential-difference equation
}

\author{
Diego Dominici* and Walter Van Assche ${ }^{\dagger}$ \\ State University of New York at New Paltz, USA \\ KU Leuven, Belgium
}

June 11, 2014

\begin{abstract}
In this paper we investigate the asymptotic distribution of the zeros of polynomials $P_{n}(x)$ satisfying a first order differential-difference equation. We give several examples of orthogonal and non-orthogonal families.

Dedicated to Frank Olver, who showed us all the asymptotic path.

MSC-class: 34E05 (Primary) 11B83, 33C45, 44A15 (Secondary)

Keywords: Differential-difference equations, polynomial sequences, Stieltjes transform,
\end{abstract} zero counting measure

\section{Introduction}

Many families of polynomials $P_{n}(x)$ satisfy differential-difference equations of the form

$$
P_{n+1}(x)=A_{n}(x) P_{n}^{\prime}(x)+B_{n}(x) P_{n}(x), \quad n \geq 0,
$$

where $P_{0}(x)=1$, and $A_{n}(x), B_{n}(x)$ are polynomials of degree at most 2 and 1 respectively

$$
\begin{aligned}
& A_{n}(x)=\alpha_{2}(n) x^{2}+\alpha_{1}(n) x+\alpha_{0}(n) \\
& B_{n}(x)=\beta_{1}(n) x+\beta_{0}(n) .
\end{aligned}
$$

Comparing powers of $x$ in (1), we see that in order to have a family of monic polynomials, we need

$$
n \alpha_{2}(n)+\beta_{1}(n)=1, \quad n \geq 0 .
$$

When $A_{n}(x)$ is independent of $n$ and $B_{n}(x)=x$, we can identify $P_{n}(x)$ with some class of derivative polynomials [16] defined by

$$
P_{n+1}(x)=Q(x) P_{n}^{\prime}(x)+x P_{n}(x),
$$

*Supported by a Humboldt Research Fellowship for experienced researchers of the Alexander von Humboldt Foundation

${ }^{\dagger}$ Supported by KU Leuven Research Project OT/12/073, FWO Project G.0934.13 and the Belgian Interuniversity Attraction Poles Programme P7/18. 
where the polynomial $Q(x)$ satisfies

$$
f^{\prime}(x)=Q[f(x)]
$$

for some function $f(x)$. These polynomials have the pseudo-Rodrigues formula

$$
P_{n}[f(x)]=\frac{1}{g(x)} \frac{d^{n}}{d x^{n}} g(x)
$$

with

$$
g(x)=\exp \left(\int^{x} f(t) d t\right)
$$

Examples of derivative polynomials include the monic Hermite polynomials $\widehat{H}_{n}(x)$, defined by [19]

$$
\widehat{H}_{n+1}(x)=-\frac{1}{2} \widehat{H}_{n}^{\prime}(x)+x \widehat{H}_{n}(x)
$$

In this case,

$$
Q(x)=-\frac{1}{2}, \quad f(x)=-\frac{1}{2} x, \quad g(x)=\exp \left(-\frac{x^{2}}{4}\right) .
$$

We will analyze the Hermite polynomials in Section 6.3.

Another example comes from taking

$$
Q(x)=x, \quad f(x)=e^{x}, \quad g(x)=\exp \left(e^{x}\right) .
$$

In this case, the polynomials $P_{n}(x)$ satisfy

$$
P_{n+1}(x)=x P_{n}^{\prime}(x)+x P_{n}(x),
$$

and are called Bell polynomials [1]. We analyze these polynomials in Section 6.4.

If

$$
Q(x)=x^{2}+1, \quad f(x)=\tan (x), \quad g(x)=\sec (x),
$$

we obtain the tangent polynomials [3]. These polynomials have applications in the theory of graded posets [15]. We analyze these polynomials in Section 6.5. The tangent polynomials are also related to the Euler-Frobenius polynomials, defined by [11]

$$
P_{n+1}(x)=\left(x^{2}-1\right) P_{n}^{\prime}(x)+x P_{n}(x) .
$$

Let $h(x)$ be analytic in a neighborhood of $x_{0}$, with $h^{\prime}\left(x_{0}\right) \neq 0$. Let $H(x)=h^{-1}(x)$ be the inverse function of $h(x)$, and suppose that

$$
f(x)=\frac{1}{h^{\prime}(x)}, \quad z_{0}=h\left(x_{0}\right), \quad f\left(x_{0}\right) \neq 0 .
$$

Then, 6]

$$
\frac{d^{n} H}{d z^{n}}\left(z_{0}\right)=\left[f\left(x_{0}\right)\right]^{n} g_{n-1}\left(x_{0}\right), \quad n=1,2, \ldots,
$$

where the functions $g_{n}(x)$ satisfy $g_{0}(x)=1$ and

$$
g_{n+1}(x)=g_{n}^{\prime}(x)+(n+1) \frac{f^{\prime}(x)}{f(x)} g_{n}(x), \quad n=0,1, \ldots
$$


If $f(x)=\exp \left(a x^{2}+b x\right)$, the functions $g_{n}(x)$ are polynomials of degree $n$. In particular, when $a=\frac{1}{2}, b=0$, we obtain a family of polynomials associated with the derivatives of the inverse error function. We analyze these polynomials in Section 6.6.

Polynomial solutions of (1) arise naturally in combinatorics as generating functions of sequences of numbers having a combinatorial interpretation. For example, the Bell polynomials are generating functions for the Stirling numbers of the second kind [29]

$$
P_{n}(x)=\sum_{k=0}^{n}\left\{\begin{array}{l}
n \\
k
\end{array}\right\} x^{k}
$$

where $\left\{\begin{array}{c}n \\ k\end{array}\right\}$ represents the number of ways to partition a set of $n$ objects into $k$ nonempty subsets.

The location of the zeros of the generating function $G(x)$ of a sequence $c_{n}$ determines some of the properties of $c_{n}$. For example, when $G(x)$ is a polynomial, we have the following result [25]:

\section{Theorem 1 Let}

$$
p(x)=c_{0}+c_{1} x+\cdots+c_{n} x^{n}
$$

be a polynomial all of whose zeros are real and negative. Then, the coefficient sequence $c_{n}$ is strictly log concave.

An extension of this result was proven by Schoenberg [24].

In this paper, we will analyze the asymptotic distribution of the zeros of polynomials defined by the differential-difference equation (1).

\section{Interlacing zeros}

In [8], we studied polynomial solutions of (1). Under some mild conditions on the coefficients $A_{n}(x), B_{n}(x)$, we concluded that in general the zeros of the polynomials $P_{n}(x)$ are real and interlace. This result would be trivial if the polynomials $P_{n}(x)$ are orthogonal but, in almost all cases, they are not.

The following theorem is crucial and of independent interest.

Theorem 2 Let $[s]$ denote the integer part of s defined by

$$
[s]=\max \{m \in \mathbb{Z}: m \leq s\} .
$$

Suppose $P_{n}(x)$ and $P_{n+1}(x)$ have interlacing zeros for every $n \in \mathbb{N}$, i.e.,

$$
x_{k, n} \leq x_{k, n-1} \leq x_{k+1, n}, \quad 1 \leq k \leq n-1
$$

and that $x_{k, n} / \phi(n) \in[a, b]$ for every $1 \leq k \leq n$ and $n \in \mathbb{N}$, where $\phi(n)$ is a positive and increasing sequence. Then, there exists an infinite subset $\Lambda \subset \mathbb{N}$ such that

$$
\lim _{n \rightarrow \infty, n \in \Lambda} \frac{\phi(n)}{n} \frac{P_{[n t]}^{\prime}(\phi(n) x)}{P_{[n t]}(\phi(n) x)}=f_{\Lambda}(t, x)
$$


for some function $f_{\Lambda}(t, x)$ which is continuous on $[0,1] \times \mathbb{C} \backslash\left[a^{*}, b^{*}\right]$, and the convergence is uniform for $t \in[0,1]$ and $x$ on compact subsets of $\mathbb{C} \backslash\left[a^{*}, b^{*}\right]$. The points $a^{*}, b^{*}$ are given by

$$
a^{*}=\min \{0, a\}, \quad b^{*}=\max \{0, b\},
$$

whenever $\phi(n)$ increases to $\infty$, and $a^{*}=a, b^{*}=b$ whenever $\phi(n)$ is constan $t^{1}$.

Proof. The partial fraction decomposition

$$
\frac{P_{m}^{\prime}(x)}{P_{m}(x)}=\sum_{k=1}^{m} \frac{1}{x-x_{k, m}}
$$

readily gives

$$
\begin{aligned}
& \phi(N)\left(\frac{P_{m}^{\prime}(\phi(N) x)}{P_{m}(\phi(N) x)}-\frac{P_{m-1}^{\prime}(\phi(N) x)}{P_{m-1}(\phi(N) x)}\right) \\
& =\sum_{k=1}^{m} \frac{1}{x-x_{k, m} / \phi(N)}-\sum_{k=1}^{m-1} \frac{1}{x-x_{k, m-1} / \phi(N)} \\
& =\frac{1}{x-x_{m, m} / \phi(N)}+\frac{1}{\phi(N)} \sum_{k=1}^{m-1} \frac{x_{k, m-1}-x_{k, m}}{\left(x-x_{k, m} / \phi(N)\right)\left(x-x_{k, m-1} / \phi(N)\right)} .
\end{aligned}
$$

If $N \geq m$ then

$$
\frac{x_{k, m}}{\phi(N)}=\frac{x_{k, m}}{\phi(m)} \frac{\phi(m)}{\phi(N)} \in\left[\frac{\phi(m)}{\phi(N)} a, \frac{\phi(m)}{\phi(N)} b\right] .
$$

Since $\phi(n)$ is increasing, we have $\phi(m) / \phi(N) \leq 1$, and if $\phi(n) \rightarrow \infty$, then

$$
\lim _{N \rightarrow \infty} \phi(m) / \phi(N)=0
$$

for every fixed $m$. This means that $x_{k, m} / \phi(N) \in\left[a^{*}, b^{*}\right]$ for every $m \leq N$. Let $K$ be a compact set in $\mathbb{C} \backslash\left[a^{*}, b^{*}\right]$ and let $\delta$ be the distance from $K$ to $\left[a^{*}, b^{*}\right]$, then $\delta>0$ and

$$
\left|x-x_{k, m} / \phi(N)\right| \geq \delta, \quad x \in K
$$

Hence

$$
\phi(N)\left|\frac{P_{m}^{\prime}(\phi(N) x)}{P_{m}(\phi(N) x)}-\frac{P_{m-1}^{\prime}(\phi(N) x)}{P_{m-1}(\phi(N) x)}\right| \leq \frac{1}{\delta}+\frac{1}{\delta^{2}} \sum_{k=1}^{m-1} \frac{x_{k, m-1}-x_{k, m}}{\phi(N)} .
$$

The interlacing (3) implies that $x_{k, m-1}-x_{k, m} \geq 0$, and furthermore $x_{k, m-1}-x_{k, m} \leq$ $x_{k+1, m}-x_{k, m}$, so that

$$
\sum_{k=1}^{m-1} \frac{x_{k, m-1}-x_{k, m}}{\phi(N)} \leq \sum_{k=1}^{m-1} \frac{x_{k+1, m}-x_{k, m}}{\phi(N)}=\frac{x_{m, m}-x_{1, m}}{\phi(N)} \leq b^{*}-a^{*}
$$

whenever $m \leq N$. This gives the bound

$$
\phi(N)\left|\frac{P_{m}^{\prime}(\phi(N) x)}{P_{m}(\phi(N) x)}-\frac{P_{m-1}^{\prime}(\phi(N) x)}{P_{m-1}(\phi(N) x)}\right| \leq \frac{1}{\delta}+\frac{b^{*}-a^{*}}{\delta^{2}}
$$

\footnotetext{
${ }^{1}$ When $\phi$ is increasing but bounded, then there is no need to introduce a scaling and one can take $\phi=1$.
} 
which holds for every $x \in K$ and every $m \leq N$. From this one easily finds

$$
\begin{aligned}
\phi(N)\left|\frac{P_{m}^{\prime}(\phi(N) x)}{P_{m}(\phi(N) x)}-\frac{P_{\ell}^{\prime}(\phi(N) x)}{P_{\ell}(\phi(N) x)}\right| & \leq \phi(N) \sum_{j=\ell+1}^{m}\left|\frac{P_{j}^{\prime}(\phi(N) x)}{P_{j}(\phi(N) x)}-\frac{P_{j-1}^{\prime}(\phi(N) x)}{P_{j-1}(\phi(N) x)}\right| \\
& \leq\left(\frac{1}{\delta}+\frac{b^{*}-a^{*}}{\delta^{2}}\right)(m-\ell)
\end{aligned}
$$

whenever $\ell \leq m \leq N$. Now take $m=[n t], \ell=[n s]$, where $0 \leq s \leq t \leq 1$, then

$$
\frac{\phi(N)}{n}\left|\frac{P_{[n t]}^{\prime}(\phi(N) x)}{P_{[n t]}(\phi(N) x)}-\frac{P_{[n s]}^{\prime}(\phi(N) x)}{P_{[n s]}(\phi(N) x)}\right| \leq C \frac{[n t]-[n s]}{n}
$$

holds for every $N \geq n$ and

$$
C=\frac{1}{\delta}+\frac{b^{*}-a^{*}}{\delta^{2}}
$$

In particular we have for $N=n$ and

$$
f_{n}(t, x)=\frac{\phi(n)}{n} \frac{P_{[n t]}^{\prime}(\phi(n) x)}{P_{[n t]}(\phi(n) x)}
$$

the inequality

$$
\left|f_{n}(t, x)-f_{n}(s, x)\right| \leq C \frac{[n t]-[n s]}{n}, \quad n \in \mathbb{N},
$$

whenever $0 \leq s \leq t \leq 1$. Let $D[0,1]$ be the space of functions $f:[0,1] \rightarrow \mathbb{C}$ that are right-continuous and have left-hand limits (see [2, Chapter 3]). In $D[0,1]$ we use the Skorohod topology and the modulus of continuity

$$
w_{f}^{\prime}(\delta)=\inf _{\left\{t_{i}\right\}} \max w_{f}\left(\left[t_{i-1}, t_{i}\right)\right),
$$

where the infimum is over all finite sets $\left\{t_{0}, t_{1}, \ldots, t_{r}\right\}$ of points in $[0,1]$ satisfying $0=$ $t_{0}<t_{i}<\cdots<t_{r}=1$ and $t_{i}-t_{i-1}>\delta$ for $1 \leq i \leq r$, and

$$
w_{f}\left(\left[t_{i-1}, t_{i}\right)\right)=\sup \left\{|f(s)-f(t)|: t_{i-1} \leq s, t<t_{i}\right\} .
$$

Observe that (7) implies that

$$
w_{f_{n}}^{\prime}(\delta) \leq \begin{cases}0 & 0 \leq \delta<1 / n \\ C / n & 1 / n \leq \delta<2 / n \\ 2 C / n & 2 / n \leq \delta<3 / n \\ \vdots & \vdots\end{cases}
$$

so that $w_{f_{n}}^{\prime}(\delta) \leq C \delta$. The analogue of the Arzelà-Ascoli theorem in $D[0,1]$ [2, Thm. 14.3] now implies that the set $\left\{f_{n}, n \in \mathbb{N}\right\}$ of functions in $D[0,1]$ has compact closure, hence there exists a subsequence $\left(f_{n}\right)_{n \in \Lambda}$ that converges in the Skorohod topology to a function $f_{\Lambda}(t, x)$ in $D[0,1]$. Convergence in the Skorohod topology does not imply pointwise convergence, but there exist strictly increasing continuous functions $\lambda_{n}:[0,1] \rightarrow[0,1]$ with $\lambda_{n}(0)=0$ and $\lambda_{n}(1)=1$, such that

$$
\lim _{n \rightarrow \infty} f_{n}\left(\lambda_{n}(t), x\right)=f_{\Lambda}(t, x)
$$


uniformly in $t$, and

$$
\lim _{n \rightarrow \infty} \lambda_{n}(t)=t
$$

uniformly in $t[2, \mathrm{p} .112]$. The inequality (7) implies that

$$
\left|f_{n}\left(\lambda_{n}(t), x\right)-f_{n}\left(\lambda_{n}(s), x\right)\right| \leq C \frac{\left[n \lambda_{n}(t)\right]-\left[n \lambda_{n}(s)\right]}{n}, \quad n \in \mathbb{N},
$$

and this gives, for $n \rightarrow \infty$,

$$
\left|f_{\Lambda}(t, x)-f_{\Lambda}(s, x)\right| \leq C(t-s),
$$

hence $f_{\Lambda}(t, x)$ is continuous, and the convergence is in fact uniform on $[0,1]$. Note that all inequalities hold uniformly for $x \in K$, hence $f_{n}(t, x)$ is a normal family on compact subsets of $\mathbb{C} \backslash\left[a^{*}, b^{*}\right]$ for every $t$ and the convergence hence also holds uniformly for $x$ on compact sets of $\mathbb{C} \backslash\left[a^{*}, b^{*}\right]$.

\section{$3 \quad$ Ratio asymptotics}

We will choose the positive increasing sequence $\phi(n)$ in such a way that the limits

$$
\begin{gathered}
\lim _{n \rightarrow \infty} \frac{n}{\phi^{2}(n)} A_{n}(\phi(n) x)=a(x), \\
\lim _{n \rightarrow \infty} \frac{1}{\phi(n)} B_{n}(\phi(n) x)=b(x)
\end{gathered}
$$

exist. Note that $a(x)$ and $b(x)$ are polynomials of degree at most 2 and 1 respectively

$$
a(z)=a_{2} z^{2}+a_{1} z+a_{0}, \quad b(z)=b_{1} z+b_{0} .
$$

The monic condition (2) implies that

$$
a_{2}+b_{1}=1 \text {. }
$$

Then, the differential-difference equation (1) gives

$$
\frac{P_{[n t]+1}(\phi(n) x)}{P_{[n t]}(\phi(n) x)}=A_{[n t]}(\phi(n) x) \frac{P_{[n t]}^{\prime}(\phi(n) x)}{\left.P_{[n t]}(\phi(n) x)\right)}+B_{[n t]}(\phi(n) x) .
$$

We will assume that $\phi(x)$ is regularly varying

$$
\phi(x)=x^{\sigma} L(x), \quad \sigma \geq 0,
$$

where $L(x)$ is slowly varying (at infinity)

$$
\lim _{n \rightarrow \infty} \frac{L(n t)}{L(n)}=1, \quad t>0 .
$$

Then, Theorem 2 and (8)-(9) imply that for the subset $\Lambda \subset \mathbb{N}$ we have

$$
\lim _{n \rightarrow \infty, n \in \Lambda} \frac{1}{\phi(n)} \frac{P_{[n t]+1}(\phi(n) x)}{P_{[n t]}(\phi(n) x)}=t^{2 \sigma-1} a\left(x t^{-\sigma}\right) f_{\Lambda}(t, x)+t^{\sigma} b\left(x t^{-\sigma}\right),
$$

which gives ratio asymptotics for the polynomials for the same subsequence where Theorem 2 gave asymptotics.

Next, we will determine an integral equation satisfied by the function $f_{\Lambda}(t, x)$. 
Theorem 3 Suppose that the polynomials $P_{n}(x)$ satisfy the differential-difference equation (1) and that

$$
\begin{aligned}
\lim _{n \rightarrow \infty} \frac{n}{\phi^{2}(n)} A_{n}(\phi(n) x) & =a(x) \\
\lim _{n \rightarrow \infty} \frac{1}{\phi(n)} B_{n}(\phi(n) x) & =b(x)
\end{aligned}
$$

where $\phi(n)=n^{\sigma} L(n)$ is regularly varying. Then, the limit function $f_{\Lambda}(s, x)$ in (4) satisfies the integral equation

$$
f_{\Lambda}(s, x)=\int_{0}^{s} \frac{\frac{d}{d x}\left[t^{2 \sigma-1} a\left(x t^{-\sigma}\right) f_{\Lambda}(t, x)+t^{\sigma} b\left(x t^{-\sigma}\right)\right]}{t^{2 \sigma-1} a\left(x t^{-\sigma}\right) f_{\Lambda}(t, x)+t^{\sigma} b\left(x t^{-\sigma}\right)} d t,
$$

with $f_{\Lambda}(0, x)=0$ and

$$
\lim _{x \rightarrow \infty} x f_{\Lambda}(s, x)=s .
$$

Proof. First we use telescopic summation to write

$$
\frac{1}{n} \frac{P_{n}^{\prime}(x)}{P_{n}(x)}=\frac{1}{n} \sum_{k=0}^{n-1}\left(\frac{P_{k+1}^{\prime}(x)}{P_{k+1}(x)}-\frac{P_{k}^{\prime}(x)}{P_{k}(x)}\right) .
$$

Observe that

$$
\begin{aligned}
\frac{P_{k+1}^{\prime}(x)}{P_{k+1}(x)}-\frac{P_{k}^{\prime}(x)}{P_{k}(x)} & =\frac{P_{k+1}^{\prime}(x) P_{k}(x)-P_{k+1}(x) P_{k}^{\prime}(x)}{P_{k+1}(x) P_{k}(x)} \\
& =\left(\frac{P_{k+1}(x)}{P_{k}(x)}\right)^{\prime} /\left(\frac{P_{k+1}(x)}{P_{k}(x)}\right)
\end{aligned}
$$

so that

$$
\frac{1}{n} \frac{P_{n}^{\prime}(x)}{P_{n}(x)}=\frac{1}{n} \sum_{k=0}^{n-1}\left(\frac{P_{k+1}(x)}{P_{k}(x)}\right)^{\prime} /\left(\frac{P_{k+1}(x)}{P_{k}(x)}\right) .
$$

This can be written as an integral

$$
\frac{1}{n} \frac{P_{n}^{\prime}(x)}{P_{n}(x)}=\int_{0}^{1}\left(\frac{P_{[n t]+1}(x)}{P_{[n t]}(x)}\right)^{\prime} /\left(\frac{P_{[n t]+1}(x)}{P_{[n t]}(x)}\right) d t .
$$

Replacing $x$ by $\phi(n) x$, we have

$$
\frac{\phi(n)}{n} \frac{P_{n}^{\prime}(\phi(n) x)}{P_{n}(\phi(n) x)}=\left.\phi(n) \int_{0}^{1} \frac{d}{d y}\left(\frac{P_{[n t]+1}(y)}{P_{[n t]}(y)}\right)\right|_{y=\phi(n) x} /\left(\frac{P_{[n t]+1}(\phi(n) x)}{P_{[n t]}(\phi(n) x)}\right) d t .
$$

If we now use Theorem 2 and $(13)$, then we find a subsequence $\Lambda \subset \mathbb{N}$ such that

$$
f_{\Lambda}(1, x)=\int_{0}^{1} \frac{\frac{d}{d x}\left(t^{2 \sigma-1} a\left(x t^{-\sigma}\right) f_{\Lambda}(t, x)+t^{\sigma} b\left(x t^{-\sigma}\right)\right)}{t^{2 \sigma-1} a\left(x t^{-\sigma}\right) f_{\Lambda}(t, x)+t^{\sigma} b\left(x t^{-\sigma}\right)} d t .
$$


In the same way, we also find

$$
\frac{\phi(n)}{n} \frac{P_{[n s]}^{\prime}(\phi(n) x)}{P_{[n s]}(\phi(n) x)}=\phi(n) \int_{0}^{[n s] / n}\left(\frac{P_{[n t]+1}(\phi(n) x)}{P_{[n t]}(\phi(n) x)}\right)^{\prime} /\left(\frac{P_{[n t]+1}(\phi(n) x)}{P_{[n t]}(\phi(n) x)}\right) d t .
$$

which for $n$ tending to infinity in $\Lambda$ gives

$$
f_{\Lambda}(s, x)=\int_{0}^{s} \frac{\frac{d}{d x}\left[t^{2 \sigma-1} a\left(x t^{-\sigma}\right) f_{\Lambda}(t, x)+t^{\sigma} b\left(x t^{-\sigma}\right)\right]}{t^{2 \sigma-1} a\left(x t^{-\sigma}\right) f_{\Lambda}(t, x)+t^{\sigma} b\left(x t^{-\sigma}\right)} d t,
$$

which is the integral-differential equation in (16). Every converging subsequence $\Lambda$ gives the same integral-differential equation. Observe that

$$
\frac{\phi(n)}{n} \frac{P_{[n t]}^{\prime}(\phi(n) x)}{P_{[n t]}(\phi(n) x)}=\frac{[n t] / n}{x}+\mathcal{O}\left(x^{-2}\right), \quad x \rightarrow \infty,
$$

hence, by letting $n \rightarrow \infty$, we have

$$
f_{\Lambda}(t, x)=\frac{t}{x}+\mathcal{O}\left(x^{-2}\right), \quad x \rightarrow \infty .
$$

\section{Abel and Riccati differential equations}

In this section we will show that the function $f_{\Lambda}(1, z)$ satisfies an ordinary differential equation (ODE) of Riccati or Abel type.

Proposition 4 We have

$$
f_{\Lambda}(t, x)=t^{1-\sigma} f_{\Lambda}\left(1, x t^{-\sigma}\right)
$$

Proof. From (4), we have

$$
f_{\Lambda}(t, x)=\lim _{\substack{n \rightarrow \infty \\ n \in \Lambda}} \frac{\phi(n)}{n} \frac{P_{[n t]}^{\prime}(\phi(n) x)}{P_{[n t]}(\phi(n) x)} .
$$

In particular, for $t=1$,

$$
f_{\Lambda}(1, x)=\lim _{\substack{n \rightarrow \infty \\ n \in \Lambda}} \frac{\phi(n)}{n} \frac{P_{n}^{\prime}(\phi(n) x)}{P_{n}(\phi(n) x)} .
$$

On the other hand,

$$
f_{\Lambda}(t, x)=\lim _{\substack{n \rightarrow \infty \\ n \in \Lambda}} \frac{\phi(n)}{\phi([n t])} \frac{[n t]}{n} \frac{\phi([n t])}{[n t]} \frac{P_{[n t]}^{\prime}(\phi(n) x)}{P_{[n t]}(\phi(n) x)}
$$

and since

$$
\lim _{n \rightarrow \infty} \frac{\phi(n)}{\phi([n t])}=\frac{1}{t^{\sigma}}, \quad \lim _{n \rightarrow \infty} \frac{[n t]}{n}=t
$$

we have

$$
f_{\Lambda}(t, x)=t^{1-\sigma} \lim _{\substack{n \rightarrow \infty \\ n \in \Lambda}} \frac{\phi([n t])}{[n t]} \frac{P_{[n t]}^{\prime}\left(\phi([n t]) t^{-\sigma} x\right)}{P_{[n t]}\left(\phi([n t]) t^{-\sigma} x\right)} .
$$

If we compare this with (19), and recall that the convergence is uniform in $x$, then

$$
f_{\Lambda}(t, x)=t^{1-\sigma} f_{\Lambda}\left(1, x t^{-\sigma}\right) .
$$


Proposition 5 For all possible $\Lambda \subset \mathbb{N}$, the function

$$
S(z)=f_{\Lambda}(1, z)
$$

satisfies the nonlinear $O D E$

$$
(1-\sigma) S(z)-\sigma z S^{\prime}(z)=\frac{a^{\prime}(z) S(z)+a(z) S^{\prime}(z)+b^{\prime}(z)}{a(z) S(z)+b(z)}
$$

with boundary condition

$$
\lim _{z \rightarrow \infty} z S(z)=1
$$

Proof. From (16) and (20), we have

$$
s^{1-\sigma} S\left(x s^{-\sigma}\right)=\int_{0}^{s} \frac{d}{d x} \ln \left[a\left(x t^{-\sigma}\right) S\left(x t^{-\sigma}\right)+b\left(x t^{-\sigma}\right)\right] d t .
$$

Differentiation with respect to $s$ gives

$$
(1-\sigma) s^{-\sigma} S\left(x s^{-\sigma}\right)-\sigma x s^{-2 \sigma} S^{\prime}\left(x s^{-\sigma}\right)=\frac{d}{d x} \ln \left[a\left(x s^{-\sigma}\right) S\left(x s^{-\sigma}\right)+b\left(x s^{-\sigma}\right)\right]
$$

or, equivalently,

$$
\begin{aligned}
& (1-\sigma) S\left(x s^{-\sigma}\right)-\sigma x s^{-\sigma} S^{\prime}\left(x s^{-\sigma}\right) \\
& =\frac{a^{\prime}\left(x s^{-\sigma}\right) S\left(x s^{-\sigma}\right)+a\left(x s^{-\sigma}\right) S^{\prime}\left(x s^{-\sigma}\right)+b^{\prime}\left(x s^{-\sigma}\right)}{a\left(x s^{-\sigma}\right) S\left(x s^{-\sigma}\right)+b\left(x s^{-\sigma}\right)} .
\end{aligned}
$$

Introducing the new variable

$$
z=x s^{-\sigma}
$$

we get

$$
(1-\sigma) S(z)-\sigma z S^{\prime}(z)=\frac{a^{\prime}(z) S(z)+a(z) S^{\prime}(z)+b^{\prime}(z)}{a(z) S(z)+b(z)} .
$$

Finally, the boundary condition 17 implies

$$
\lim _{z \rightarrow \infty} z S(z)=1 .
$$

Corollary 6 The function $S(z)$ satisfies the integral equation

$$
\int^{z} S(x) d x+\sigma[1-z S(z)]=\ln [a(z) S(z)+b(z)] .
$$

Proof. Integrating 21], we have

$$
\int^{z} S(x) d x+C-\sigma z S(z)=\ln [a(z) S(z)+b(z)],
$$


for some constant $C$. From 22, we obtain

$$
\int^{z} S(x) d x \sim \ln (z), \quad z \rightarrow \infty
$$

and

$$
a(z) S(z)+b(z) \sim\left(a_{2}+b_{1}\right) z=z, \quad z \rightarrow \infty
$$

Thus, $C=\sigma$.

Solving for $S^{\prime}(z)$ in $(21)$, we get

$$
[z \sigma(a S+b)+a] S^{\prime}=(1-\sigma) S(a S+b)-\left(b^{\prime}+S a^{\prime}\right) .
$$

If $\sigma=0$, then

$$
S^{\prime}=S^{2}+\frac{b(z)-a^{\prime}(z)}{a(z)} S-\frac{b^{\prime}(z)}{a(z)} .
$$

Thus, in this case $S(z)$ is the solution of a Riccati equation [23]. The substitution

$$
u(z)=\exp \left[-\int^{z} S(t) d t\right]
$$

reduces 24 to a second-order linear ODE

$$
a(z) u^{\prime \prime}+\left[a^{\prime}(z)-b(z)\right] u^{\prime}-b^{\prime}(z) u=0
$$

which can be rewritten as

$$
\left[a(z) u^{\prime}-b(z) u\right]^{\prime}=0
$$

Thus,

$$
a(z) u^{\prime}-b(z) u=C_{1}
$$

and therefore

$$
u(z)=\frac{1}{h(z)}\left[C_{1} \int^{z} \frac{h(t)}{a(t)} d t+C_{2}\right]
$$

where

$$
h(z)=\exp \left[-\int^{z} \frac{b(t)}{a(t)} d t\right]
$$

Using (26) in 25), we get

$$
S(z)=-\frac{d}{d z} \ln [u(z)]=-\frac{b(z)}{a(z)}-\frac{h(z)}{a(z)}\left[\int^{z} \frac{h(t)}{a(t)} d t+C\right]^{-1} .
$$

Alternatively, we note that

$$
S_{p}(z)=-\frac{b(z)}{a(z)}
$$


is a particular solution of the Riccati equation (24). Thus, we can set [22]

$$
S(z)=S_{p}(z)+\frac{1}{y(z)}
$$

in (24) and obtain the linear equation

$$
y^{\prime}-\frac{a^{\prime}+b}{a} y=-1
$$

with solution

$$
y(z)=-u(z)\left[\int^{z} \frac{1}{u(t)} d t+C\right]
$$

where

$$
u(z)=\exp \left[\int^{z} \frac{a^{\prime}(t)+b(t)}{a(t)} d t\right]=a(z) \exp \left[\int^{z} \frac{b(t)}{a(t)} d t\right] .
$$

Using (31) in (29), we recover (27).

If $\sigma \neq 0$, we have

$$
[S+g(z)] S^{\prime}=f_{2}(z) S^{2}+f_{1}(z) S+f_{0}(z)
$$

where

$$
\begin{aligned}
& g(z)=\frac{a(z)+\sigma z b(z)}{\sigma z a(z)}, \quad f_{0}(z)=-\frac{b^{\prime}(z)}{\sigma z a(z)}, \\
& f_{1}(z)=\frac{(1-\sigma) b(z)-a^{\prime}(z)}{\sigma z a(z)}, \quad f_{2}(z)=\frac{1-\sigma}{\sigma z} .
\end{aligned}
$$

Differential equations of the form $(32)$ are called Abel equations of the second kind [18]. The substitution

$$
w(z)=[S(z)+g(z)] E(z)
$$

where

$$
E(z)=\exp \left[-\int^{z} f_{2}(t) d t\right]=z^{1-\sigma^{-1}}
$$

transforms equation 32 to the canonical form

$$
w\left(\frac{d w}{d x}-1\right)=R(x)
$$

where

$$
R(z)=\frac{F_{0}(z)}{F_{1}(z)}
$$

with

$$
\begin{aligned}
& F_{0}(z)=\left[f_{0}(z)-f_{1}(z) g(z)+f_{2}(z) g^{2}(z)\right] E^{2}(z) \\
& F_{1}(z)=\left[f_{1}(z)-2 f_{2}(z) g(z)+g^{\prime}(z)\right] E(z)
\end{aligned}
$$


and the new variable $x$ is defined by

$$
x=\int^{z} F_{1}(t) d t
$$

General solutions of (36) for different functions $R(x)$ are given in [22].

Once again, we note that $(28)$ is a particular solution of the Abel equation (23). With this in mind, we can rewrite equation 34 in the form

$$
S(z)=S_{p}(z)-\frac{1}{\sigma z}+z^{\frac{1}{\sigma}-1} w(z)
$$

We can also use the particular solution 28 to construct a self-transformation of the Abel equation (32). Setting

$$
y(z)=\frac{1}{S(z)-S_{p}(z)}
$$

we get 22

$$
\left(y+\frac{1}{S_{p}+g}\right) y^{\prime}=\frac{S_{p}^{\prime}-f_{1}-2 f_{2} S_{p}}{S_{p}+g} y^{2}-\frac{f_{2}}{S_{p}+g} y .
$$

From 33 we have

$$
S_{p}+g=\frac{a(z)+\sigma z b(z)}{\sigma z a(z)}-\frac{b(z)}{a(z)}=\frac{1}{\sigma z}
$$

and

$$
\frac{S_{p}^{\prime}-f_{1}-2 f_{2} S_{p}}{S_{p}+g}=\sigma z S_{p}^{\prime}+(\sigma-1) S_{p}+\frac{a^{\prime}}{a}
$$

Thus, we obtain the equation

$$
(y+\sigma z) y^{\prime}=\left[\sigma z S_{p}^{\prime}+(\sigma-1) S_{p}+\frac{a^{\prime}}{a}\right] y^{2}+(\sigma-1) y .
$$

When $\sigma=0$, equation (39) reduces to the Riccati equation (30).

Since there is no method that will allow us to solve the Abel equation (23) in general, we will construct a particular solution satisfying the asymptotic condition (22).

Proposition 7 Suppose that

$$
\left(a_{2}+\sigma\right) n+1 \neq 0, \quad n=1,2, \ldots
$$

Then, the Abel equation

$$
[z \sigma(a S+b)+a] S^{\prime}=(1-\sigma) S(a S+b)-\left(b^{\prime}+S a^{\prime}\right)
$$

with the asymptotic condition

$$
\lim _{z \rightarrow \infty} z S(z)=1
$$

has the unique solution

$$
S(z)=\sum_{n=0}^{\infty} \frac{c_{n}}{z^{n}}
$$


where the coefficients $c_{n}$ are defined by the recurrence relation

$$
\begin{gathered}
{\left[\left(a_{2}+\sigma\right) n+1\right] c_{n}=\left[(2 \sigma-1-\sigma n)\left(a_{1}+b_{0}\right)-a_{1}(n-2)\right] c_{n-1}} \\
-a_{0}(n-2) c_{n-2}-\sum_{k=0}^{n-3}(\sigma k+1) c_{k+1}\left(a_{0} c_{n-2-k}+a_{1} c_{n-1-k}\right)+a_{2}(\sigma k+\sigma+1) c_{k+2} c_{n-1-k},
\end{gathered}
$$

for $n=2,3, \ldots$, with $c_{0}=0$ and $c_{1}=1$.

Proof. Setting $z=x^{-1}$ in $(23)$, we have

$$
-x^{2}\left[\sigma x^{-1}(a S+b)+a\right] S^{\prime}=(1-\sigma) S(a S+b)-\left(b^{\prime}+S a^{\prime}\right),
$$

where $a, b, a^{\prime}$ and $b^{\prime}$ are evaluated at $x^{-1}$. Using $(10)$ and 40 in $(42)$, we obtain

$$
\begin{gathered}
-b_{1} x^{2}+a_{0} \sum_{n=3}^{\infty}(n-3) c_{n-3} x^{n} \\
+\sum_{n=2}^{\infty}\left[(n-2)\left(\sigma b_{0}+a_{1}\right)-\left(\sigma b_{0}-b_{0}+a_{1}\right)\right] c_{n-2} x^{n} \\
+\sum_{n=1}^{\infty}\left[(n-1)\left(\sigma b_{1}+a_{2}\right)-\left(\sigma b_{1}-b_{1}+2 a_{2}\right)\right] c_{n-1} x^{n} \\
+a_{0} \sum_{n=2}^{\infty} \sum_{k=2}^{n}[\sigma(k-2)-(\sigma-1)] c_{k-2} c_{n-k} x^{n} \\
+a_{1} \sum_{n=1}^{\infty} \sum_{k=1}^{n}[\sigma(k-1)-(\sigma-1)] c_{k-1} c_{n-k} x^{n} \\
+a_{2} \sum_{n=0}^{\infty} \sum_{k=0}^{n}[\sigma k-(\sigma-1)] c_{k} c_{n-k} x^{n}=0 .
\end{gathered}
$$

Comparing coefficients of $x$ we get for $x^{0}$ and $x^{1}$

$$
\begin{array}{r}
a_{2}(1-\sigma) c_{0}^{2}=0, \\
a_{2}(2-\sigma) c_{0} c_{1}-\left(\sigma b_{1}-b_{1}+2 a_{2}\right) c_{0}-a_{1}(\sigma-1) c_{0}^{2}=0,
\end{array}
$$

and therefore $c_{0}=0$. The next equation is

$$
-b_{1}+\left(b_{1}-a_{2}\right) c_{1}+a_{2} c_{1}^{2}=0
$$

or

$$
\left(c_{1}-1\right)\left(b_{1}+a_{2} c_{1}\right)=0,
$$

and hence, $c_{1}=1$ because of $(22)$. Using $c_{0}=0$ in (43), we get after some simplification

$$
\begin{gathered}
{\left[(n-2)\left(\sigma b_{0}+a_{1}\right)-\left(\sigma b_{0}-b_{0}+a_{1}\right)\right] c_{n-2}} \\
+\left[(n-1)\left(\sigma b_{1}+a_{2}\right)-\left(\sigma b_{1}-b_{1}+2 a_{2}\right)\right] c_{n-1} \\
+a_{0} \sum_{k=3}^{n-1}[\sigma(k-3)+1] c_{k-2} c_{n-k}+a_{1} \sum_{k=2}^{n-1}[\sigma(k-2)+1] c_{k-1} c_{n-k} \\
+a_{0}(n-3) c_{n-3}+a_{2} \sum_{k=1}^{n-1}[\sigma(k-1)+1] c_{k} c_{n-k}=0 .
\end{gathered}
$$


Shifting $n$ to $n+1$, we have

$$
\begin{gathered}
{\left[(n-1)\left(\sigma b_{0}+a_{1}\right)-\left(\sigma b_{0}-b_{0}+a_{1}\right)\right] c_{n-1}} \\
+\left[n\left(\sigma b_{1}+a_{2}\right)-\left(\sigma b_{1}-b_{1}+2 a_{2}\right)\right] c_{n} \\
+a_{0} \sum_{k=3}^{n}[\sigma(k-3)+1] c_{k-2} c_{n+1-k}+a_{1} \sum_{k=2}^{n}[\sigma(k-2)+1] c_{k-1} c_{n+1-k} \\
+a_{0}(n-2) c_{n-2}+a_{2} \sum_{k=1}^{n}[\sigma(k-1)+1] c_{k} c_{n+1-k}=0 .
\end{gathered}
$$

Using $c_{1}=1$ in 44 , we obtain

$$
\begin{gathered}
{\left[(n-1)\left(\sigma b_{0}+a_{1}\right)-\left(\sigma b_{0}-b_{0}+a_{1}\right)+a_{1}(n \sigma-2 \sigma+1)\right] c_{n-1}} \\
+\left[n\left(\sigma b_{1}+a_{2}\right)-\left(\sigma b_{1}-b_{1}+2 a_{2}\right)+a_{2}(n \sigma-\sigma+2)\right] c_{n} \\
+a_{0} \sum_{k=3}^{n}[\sigma(k-3)+1] c_{k-2} c_{n+1-k}+a_{1} \sum_{k=2}^{n-1}[\sigma(k-2)+1] c_{k-1} c_{n+1-k} \\
+a_{0}(n-2) c_{n-2}+a_{2} \sum_{k=2}^{n-1}[\sigma(k-1)+1] c_{k} c_{n+1-k}=0 .
\end{gathered}
$$

Shifting the sums in 45 we conclude that

$$
\begin{aligned}
& {\left[(n-1)\left(\sigma b_{0}+a_{1}\right)-\left(\sigma b_{0}-b_{0}+a_{1}\right)+a_{1}(n \sigma-2 \sigma+1)\right] c_{n-1}} \\
& \quad+\left[n\left(\sigma b_{1}+a_{2}\right)-\left(\sigma b_{1}-b_{1}+2 a_{2}\right)+a_{2}(n \sigma-\sigma+2)\right] c_{n} \\
& +a_{0} \sum_{k=0}^{n-3}(\sigma k+1) c_{k+1} c_{n-2-k}+a_{1} \sum_{k=0}^{n-3}(\sigma k+1) c_{k+1} c_{n-1-k} \\
& \quad+a_{0}(n-2) c_{n-2}+a_{2} \sum_{k=0}^{n-3}[\sigma(k+1)+1] c_{k+2} c_{n-1-k}=0
\end{aligned}
$$

and using (11), the result follows.

Corollary 8 Under the conditions of Theorem 2, the limit function $f(t, x)$

$$
f(t, x)=\lim _{n \rightarrow \infty, n \in \Lambda} \frac{\phi(n)}{n} \frac{P_{[n t]}^{\prime}(\phi(n) x)}{P_{[n t]}(\phi(n) x)}
$$

is independent of the subsequence $\Lambda$. Moreover, we have

$$
f(t, x)=t^{1-\sigma} S\left(x t^{-\sigma}\right),
$$

where $S(z)$ satisfies the differential equation (21) and the boundary condition (22).

\section{The Stieltjes transform}

We will now show that the function $S(z)$ that we obtained in the previous section is the Stieltjes transform of the asymptotic zero distribution for the polynomials $P_{n}(x)$. 
From (46), (6) and (20), we know that

$$
S(z)=f(1, z)=\lim _{n \rightarrow \infty} \frac{\phi(n)}{n} \frac{P_{[n t]}^{\prime}(\phi(n) x)}{P_{[n t]}(\phi(n) x)}=\lim _{n \rightarrow \infty} \frac{1}{n} \sum_{k=1}^{n} \frac{1}{z-z_{k, n}},
$$

where

$$
z_{k, n}=\frac{x_{k, n}}{\phi(n)},
$$

and $\left\{x_{k, n}\right\}_{k=1}^{n}$ are the zeros of $P_{n}(x)$. Introducing the zero counting distributions (and corresponding zero counting measures) [27, Section 1.2]

$$
\psi_{n}(t)=\left\{\begin{array}{c}
0, \quad t \leq z_{1, n} \\
\frac{k}{n}, \quad z_{k, n}<t \leq z_{k+1, n}, \quad k=1,2, \ldots, n-1 \\
1, \quad t \geq z_{n, n}
\end{array},\right.
$$

we have

$$
\frac{1}{n} \sum_{k=1}^{n} \frac{1}{z-z_{k, n}}=\int_{-\infty}^{\infty} \frac{1}{z-t} d \psi_{n}(t)
$$

and there exists a probability distribution $\psi(t)$ such that

$$
S(z)=\int_{-\infty}^{\infty} \frac{1}{z-t} d \psi(t)
$$

The integral above is called the Stieltjes transform [28, Section 65] of $\psi(t)$, and $\psi(t)$ is called the asymptotic zero distribution. To recover the distribution function $\psi(t)$ from (48), we can use the Stieltjes-Perron inversion formula

$$
[\psi](s)-[\psi](t)=\frac{1}{\pi} \lim _{y \rightarrow 0^{+}} \int_{s}^{t} \operatorname{Im} S(x+\mathrm{i} y) d x,
$$

where $[\psi]$ denotes the jump operator

$$
[\psi](s)=\lim _{\varepsilon \rightarrow 0^{+}} \frac{\psi(s+\varepsilon)+\psi(s-\varepsilon)}{2} .
$$

The absolutely continuous part of $\psi$ is given by

$$
\psi^{\prime}(t)=-\frac{1}{\pi} \lim _{y \rightarrow 0^{+}} \operatorname{Im} S(t+\mathrm{i} y) .
$$

The function $S(z)$ has the asymptotic behavior [14, Section 12.9]

$$
S(z) \sim \frac{\mu_{0}}{z}+\frac{\mu_{1}}{z^{2}}+\frac{\mu_{2}}{z^{3}}+\cdots, \quad z \rightarrow \infty,
$$

where the coefficients $\mu_{n}$ are the moments of the distribution function $\psi(t)$

$$
\mu_{n}=\int_{-\infty}^{\infty} t^{n} d \psi(t), \quad n=0,1, \ldots
$$

Remark 9 Note that Stieltjes transforms satisfying a Riccati equation have also been considered in [20]. 


\section{Examples}

\subsection{Jacobi polynomials}

The Jacobi polynomials $P_{n}^{(\alpha, \beta)}(x)$ are orthogonal polynomials on $[-1,1]$ satisfying

$\int_{-1}^{1} P_{n}^{(\alpha, \beta)}(x) P_{m}^{(\alpha, \beta)}(x)(1-x)^{\alpha}(1+x)^{\beta} d x=\frac{2^{\alpha+\beta+1}}{2 n+\alpha+\beta+1} \frac{\Gamma(n+\alpha+1) \Gamma(n+\beta+1)}{\Gamma(n+\alpha+\beta+1) n !} \delta_{m, n}$,

where we take $\alpha, \beta>-1$ in order that the weight is integrable on $[-1,1]$. They satisfy the relation [26, Eq. (4.5.7) on p. 72]

$(2 n+\alpha+\beta+2)\left(1-x^{2}\right) \frac{d}{d x} P_{n}^{(\alpha, \beta)}(x)=(n+\alpha+\beta+1)[(2 n+\alpha+\beta+2) x+\alpha-\beta] P_{n}^{(\alpha, \beta)}(x)$

$$
-2(n+1)(n+\alpha+\beta+1) P_{n+1}^{(\alpha, \beta)}(x) .
$$

The monic polynomials are

$$
P_{n}(x)=\frac{2^{n} n ! \Gamma(n+\alpha+\beta+1)}{\Gamma(2 n+\alpha+\beta+1)} P_{n}^{(\alpha, \beta)}(x)
$$

and the relation (51) becomes

$$
(2 n+\alpha+\beta+1) P_{n+1}(x)=\left(x^{2}-1\right) P_{n}^{\prime}(x)+(n+\alpha+\beta+1)\left(x+\frac{\alpha-\beta}{2 n+\alpha+\beta+2}\right) P_{n}(x) .
$$

This is of the form (1) with

$$
A_{n}(x)=\frac{x^{2}-1}{2 n+\alpha+\beta+1}, \quad B_{n}(x)=\frac{n+\alpha+\beta+1}{2 n+\alpha+\beta+1}\left(x+\frac{\alpha-\beta}{2 n+\alpha+\beta+2}\right) .
$$

All the zeros of Jacobi polynomials are on $[-1,1]$ and they are interlacing. Hence we need no scaling and can take $\phi(n)=1$ for all $n$. Clearly $\sigma=0$ and

$$
\begin{aligned}
\lim _{n \rightarrow \infty} n A_{n}(x) & =\frac{1}{2}\left(x^{2}-1\right)=a(x), \\
\lim _{n \rightarrow \infty} B_{n}(x) & =\frac{x}{2}=b(x) .
\end{aligned}
$$

It follows that

$$
h(x)=\exp \left[-\int^{x} \frac{t}{t^{2}-1} d t\right]=\frac{1}{\sqrt{x^{2}-1}} .
$$

Using (53) and (54) in (27), we get

$$
S(x)=-\frac{x}{x^{2}-1}+\frac{2}{x^{2}-1} \frac{1}{x+C \sqrt{x^{2}-1}} .
$$


In order that $\lim _{x \rightarrow \infty} x S(x)=1$ we need to choose $C=-1$, which gives

$$
f(1, x)=S(x)=\frac{1}{\sqrt{x^{2}-1}} .
$$

This function is analytic in $\mathbb{C} \backslash[-1,1]$ and is the Stieltjes transform of a positive measure (the arcsin distribution):

$$
\frac{1}{\sqrt{x^{2}-1}}=\frac{1}{\pi} \int_{-1}^{1} \frac{1}{x-t} \frac{d t}{\sqrt{1-t^{2}}}, \quad x \notin[-1,1],
$$

so that the asymptotic distribution of the zeros of Jacobi polynomials is given by

$$
\lim _{n \rightarrow \infty} \frac{1}{n} \#\left\{\text { zeros of } P_{n}^{(\alpha, \beta)} \text { in }[a, b]\right\}=\frac{1}{\pi} \int_{a}^{b} \frac{d t}{\sqrt{1-t^{2}}}, \quad[a, b] \subset[-1,1] .
$$

This result is well known and can be found in many ways: by using asymptotic formulas of the Jacobi polynomials, logarithmic potential theory or the three term recurrence relation. Our approach using Theorem 3 is new.

\subsection{Laguerre polynomials}

Laguerre polynomials $L_{n}^{\alpha}(x)$ are orthogonal polynomials on $[0, \infty)$

$$
\int_{0}^{\infty} L_{n}^{\alpha}(x) L_{m}^{\alpha}(x) x^{\alpha} e^{-x} d x=\frac{\Gamma(n+\alpha+1)}{n !} \delta_{m, n}
$$

where $\alpha>-1$. The zeros are real, positive and interlacing. From Szegő [26, Eq. (5.1.14)] we learn that

$$
x \frac{d}{d x} L_{n}^{\alpha}(x)=n L_{n}^{\alpha}(x)-(n+\alpha) L_{n-1}^{\alpha}(x) .
$$

Combined with the recurrence relation [26, Eq. (5.1.10)]

$$
(n+1) L_{n+1}^{\alpha}(x)=(-x+2 n+\alpha+1) L_{n}^{\alpha}(x)-(n+\alpha) L_{n-1}^{\alpha}(x)
$$

this gives the relation

$$
x \frac{d}{d x} L_{n}^{\alpha}(x)=(x-n-\alpha-1) L_{n}^{\alpha}(x)+(n+1) L_{n+1}^{\alpha}(x) .
$$

For the monic polynomials $P_{n}=(-1)^{n} n ! L_{n}^{\alpha}$ we then find

$$
P_{n+1}(x)=-x P_{n}^{\prime}(x)+(x-n-\alpha-1) P_{n}(x),
$$

which is of the form (1) with

$$
A_{n}(x)=-x, \quad B_{n}(x)=x-n-\alpha-1 .
$$


In order that (14)-(15) holds, we choose the scaling $\phi(n)=n$, so that

$$
a(x)=-x, \quad b(x)=x-1
$$

and $\sigma=1$. Using these in (34), (35), (37) and (38), we have

$$
w(z)=S(z)-1+\frac{2}{z}, \quad E(z)=1, \quad R(z)=-\frac{2}{3 z}, \quad x=\frac{3}{z} .
$$

Hence, $R(x)=-\frac{2}{9} x$ and the canonical form of the Abel equation 36 is

$$
w\left(\frac{d w}{d x}-1\right)=-\frac{2}{9} x
$$

and therefore

$$
w(x)=\frac{2 C x+1 \pm \sqrt{2 C x+1}}{3 C}
$$

Using (57), we get

$$
S(z)=\frac{3 C+1 \pm \sqrt{6 C z^{-1}+1}}{3 C}
$$

which gives

$$
0=\lim _{z \rightarrow \infty} S(z)=\frac{3 C+1 \pm 1}{3 C} .
$$

Thus, the desired solution has the positive sign and $C=-\frac{2}{3}$

$$
f(1, z)=S(z)=\frac{1-\sqrt{1-4 z^{-1}}}{2}=\frac{2}{z+\sqrt{z^{2}-4 z}} .
$$

This is the Stieltjes transform of a positive measure on $[0,4]$ (the Marchenko-Pastur distribution)

$$
\frac{1}{2 \pi} \int_{0}^{4} \frac{\sqrt{4-t}}{\sqrt{t}} \frac{d t}{z-t}=\frac{2}{z+\sqrt{z^{2}-4 z}}, \quad z \notin[0,4]
$$

so that we can conclude

$$
\lim _{n \rightarrow \infty} \frac{1}{n} \#\left\{\text { zeros of } L_{n}^{\alpha}(n x) \text { in }[a, b]\right\}=\frac{1}{2 \pi} \int_{a}^{b} \frac{\sqrt{4-t}}{\sqrt{t}} d t, \quad[a, b] \subset[0,4] .
$$

This result is not new and can be found using general methods based on potential theory or general results for orthogonal polynomials defined by their recurrence relation. The approach using Theorem 3 is new.

\subsection{Hermite polynomials}

The monic Hermite polynomials $H_{n}(x)$ are orthogonal on the real line with respect to the normal distribution

$$
\int_{-\infty}^{\infty} H_{n}(x) H_{m}(x) e^{-x^{2}} d x=\frac{\sqrt{\pi} n !}{2^{n}} \delta_{m, n}
$$


They satisfy the following differential-difference equation [26, Eq. (5.5.10)]

$$
H_{n+1}(x)=-\frac{1}{2} H_{n}^{\prime}(x)+x H_{n}(x) .
$$

The choice $\phi(n)=\sqrt{n}$ gives a scaling such that $(14)-(15)$ result in

$$
a(x)=-\frac{1}{2}, \quad b(x)=x
$$

Using (58) and $\sigma=1 / 2$ in (34), (35), (37) and (38), we have

$$
w(z)=\frac{1}{z} S(z)-2+\frac{2}{z^{2}}, \quad E(z)=\frac{1}{z}, \quad R(z)=-\frac{2}{3 z^{2}}, \quad x=\frac{3}{z^{2}} .
$$

Hence, $R(x)=-\frac{2}{9} x$ and the canonical form of the Abel equation (36) is the same as the one for the Laguerre polynomials

$$
w\left(\frac{d w}{d x}-1\right)=-\frac{2}{9} x
$$

and therefore

$$
S(z)=\frac{6 C z^{-2}+1 \pm \sqrt{6 C z^{-2}+1}}{3 C} z+2 z-\frac{2}{z} .
$$

This behaves for $z \rightarrow \infty$ as

$$
\frac{1 \pm 1}{3 C} z+2 z
$$

hence the required solution corresponds to the positive sign and $C=-\frac{1}{3}$, giving

$$
S(z)=\left(1-\sqrt{1-2 z^{-2}}\right) z=\frac{2}{z+\sqrt{z^{2}-2}} .
$$

This is the Stieltjes transform of a measure on $[-\sqrt{2}, \sqrt{2}]$

$$
\frac{2}{z+\sqrt{z^{2}-2}}=\frac{1}{\pi} \int_{-\sqrt{2}}^{\sqrt{2}} \frac{\sqrt{2-t^{2}}}{z-t} d t, \quad z \notin[-\sqrt{2}, \sqrt{2}]
$$

and consequently

$$
\lim _{n \rightarrow \infty} \frac{1}{n} \#\left\{\text { zeros of } H_{n}(\sqrt{n} x) \text { in }[a, b]\right\}=\frac{1}{\pi} \int_{a}^{b} \sqrt{2-t^{2}} d t, \quad[a, b] \subset[-\sqrt{2}, \sqrt{2}] .
$$

Again, this result is not new and corresponds to the famous semi-circle law for the eigenvalues of random matrices. 


\subsection{Bell polynomials}

The Bell polynomials $P_{n}(x)$ satisfy the equation [1]

$$
P_{n+1}(x)=x P_{n}^{\prime}(x)+x P_{n}(x) .
$$

In [7, we obtained asymptotic approximations for these polynomials using a discrete version of the ray method. Choosing $\phi(n)=n$, we get

$$
a(z)=z, \quad b(z)=z .
$$

Using (60) and $\sigma=1$ in (34), (35), 37) and (38), we have

$$
w(z)=S(z)+1+\frac{1}{z}, \quad E(z)=1, \quad R(z)=-\frac{1}{2 z}, \quad x=\frac{2}{z} .
$$

Hence, $R(x)=-\frac{1}{4} x$ and the canonical form of the Abel equation 36 is

$$
w\left(\frac{d w}{d x}-1\right)=-\frac{1}{4} x
$$

and therefore

$$
w(x)=\frac{x}{2}\left[1+\frac{1}{\mathrm{~W}(C x)}\right]
$$

where $\mathrm{W}(z)$ is the principal branch of the Lambert W function [4] defined by

$$
z=\mathrm{W}(z) e^{\mathrm{W}(z)}
$$

which is the branch that maps the positive real axis onto itself. The function $W(z)$ is discontinuous on the branch cut $B=\left(-\infty,-e^{-1}\right)$. On the cut plane $\mathbb{C} \backslash B$, the function is holomorphic. Its real values obey $\mathrm{W}(0)=0$ and

$$
\begin{aligned}
& \mathrm{W}(x) \in[-1,0), \quad x \in\left[-e^{-1}, 0\right) \\
& \mathrm{W}(x)>0, \quad x>0 .
\end{aligned}
$$

The imaginary part of $\mathrm{W}(x)$ has the following range of values for real $x$

$$
\operatorname{Im} \mathrm{W}(x) \in(0, \pi), \quad x \in\left(-\infty,-e^{-1}\right)
$$

and $\operatorname{Im} W(x)=0$ otherwise. $\operatorname{Im} W(z)$ and $\operatorname{Im}(z)$ have the same sign in the cut plane $\mathbb{C} \backslash \mathbb{R}$

$$
\operatorname{Im} \mathrm{W}(z) \operatorname{Im}(z)>0, \quad z \notin \mathbb{R}
$$

We also have

$$
\lim _{y \rightarrow 0^{+}} \operatorname{Im} \mathrm{W}(x+\mathrm{i} y)=\operatorname{Im} \mathrm{W}(x), \quad x \in \mathbb{R} .
$$

Using 61) in 62, we obtain

$$
S(z)=\left[z \mathrm{~W}\left(\frac{2 C}{z}\right)\right]^{-1}-1
$$


The Taylor series of the function $\mathrm{W}(z)$ around 0 is given by [5]

$$
\mathrm{W}(z)=\sum_{n=1}^{\infty}(-n)^{n-1} \frac{z^{n}}{n !}, \quad|z|<e^{-1}
$$

Using 66 in (65), we get

$$
S(z) \sim \frac{1}{2 C}-1+\frac{1}{z}, \quad z \rightarrow \infty
$$

Thus, we need $C=\frac{1}{2}$ and conclude that

$$
S(z)=\left[z \mathrm{~W}\left(\frac{1}{z}\right)\right]^{-1}-1=e^{\mathrm{W}\left(\frac{1}{z}\right)}-1 .
$$

Applying the Lagrange Inversion Formula [29] to (63), we have

$$
e^{\mathrm{W}(z)}=\sum_{n=0}^{\infty}(1-n)^{n-1} \frac{z^{n}}{n !}, \quad|z|<e^{-1} .
$$

Hence,

$$
S(z)=e^{\mathrm{W}\left(\frac{1}{z}\right)}-1=\sum_{n=0}^{\infty} \frac{(-n)^{n}}{(n+1) !} \frac{1}{z^{n+1}}, \quad|z|>e .
$$

The function $S(z)$ has a branch cut along the interval [ $-e, 0]$.Using the results in [17], we also have the integral representation

$$
S(z)=\frac{1}{\pi} \int_{0}^{\pi} \frac{v^{2}+[1-v \cot (v)]^{2}}{v^{2} \csc ^{2}(v)\left[z+v^{-1} \sin (v) e^{v \cot (v)}\right]} d v, \quad|\arg (z)|<\pi .
$$

From (49) and (64) we have, for $t \in(-e, 0)$

$$
\psi^{\prime}(t)=-\frac{1}{\pi} \lim _{y \rightarrow 0^{+}} \operatorname{Im} \exp \left[\mathrm{W}\left(\frac{1}{t+\mathrm{i} y}\right)\right]=\frac{1}{\pi} \operatorname{Im} \exp \left[\mathrm{W}\left(\frac{1}{t}\right)\right] .
$$

In [13], C. Elbert studied the zero asymptotics of $P_{n}(x)$, and obtained

$$
\psi(t)=1+\frac{1}{\pi}\left[\operatorname{Im} \frac{1}{\mathrm{~W}\left(\frac{1}{t}\right)}-\arg \mathrm{W}\left(\frac{1}{t}\right)\right], \quad t \in(-e, 0),
$$

although he didn't identify the function appearing in his formulas with the Lambert W function. His method was completely different, and was based on his previous work on the asymptotic analysis of $P_{n}(x)$ using the saddle point method [12].

We conclude that

$$
\lim _{n \rightarrow \infty} \frac{1}{n} \#\left\{\text { zeros of } P_{n}(n x) \text { in }[a, b]\right\}=\frac{1}{\pi} \int_{a}^{b} \operatorname{Im} \exp \left[\mathrm{W}\left(\frac{1}{t}\right)\right] d t, \quad[a, b] \subset[-e, 0] .
$$

In Figure 1 we plot the zero counting distribution $\psi_{75}(t)$ defined in (47) and the distribution function $\psi(t)$ defined in $(67)$, to illustrate the accuracy of our results. 


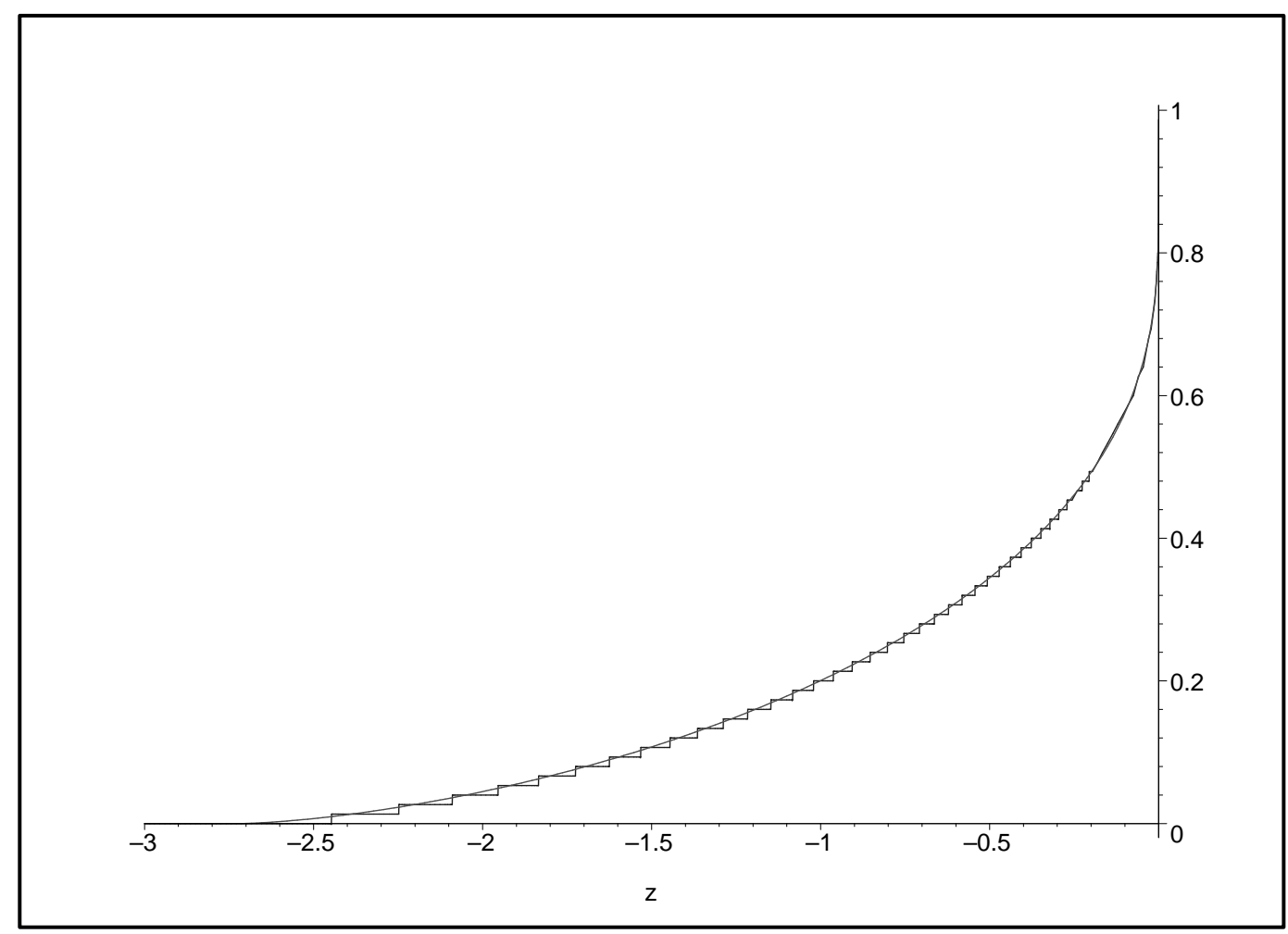

Figure 1: A plot of $\psi_{75}$ (step function) and $\psi$ (solid line) for the Bell polynomials

\subsection{Tangent polynomials}

The tangent polynomials $P_{n}(x)$ are defined by $P_{0}(x)=1$ and

$$
P_{n+1}(x)=\left(x^{2}+1\right) P_{n}^{\prime}(x)+x P_{n}(x) .
$$

Since they have purely imaginary zeros, we set

$$
Q_{n}(x)=\frac{(\mathrm{i})^{n}}{n !} P_{n}(-\mathrm{i} x),
$$

and obtain a family of monic polynomials with real zeros, satisfying

$$
Q_{n+1}(x)=\frac{x^{2}-1}{n+1} Q_{n}^{\prime}(x)+\frac{x}{n+1} Q_{n}(x) .
$$

Choosing $\phi(n)=1$, we get

$$
\lim _{n \rightarrow \infty} n A_{n}(x)=x^{2}-1=a(x), \quad \lim _{n \rightarrow \infty} B_{n}(x)=0=b(x) .
$$




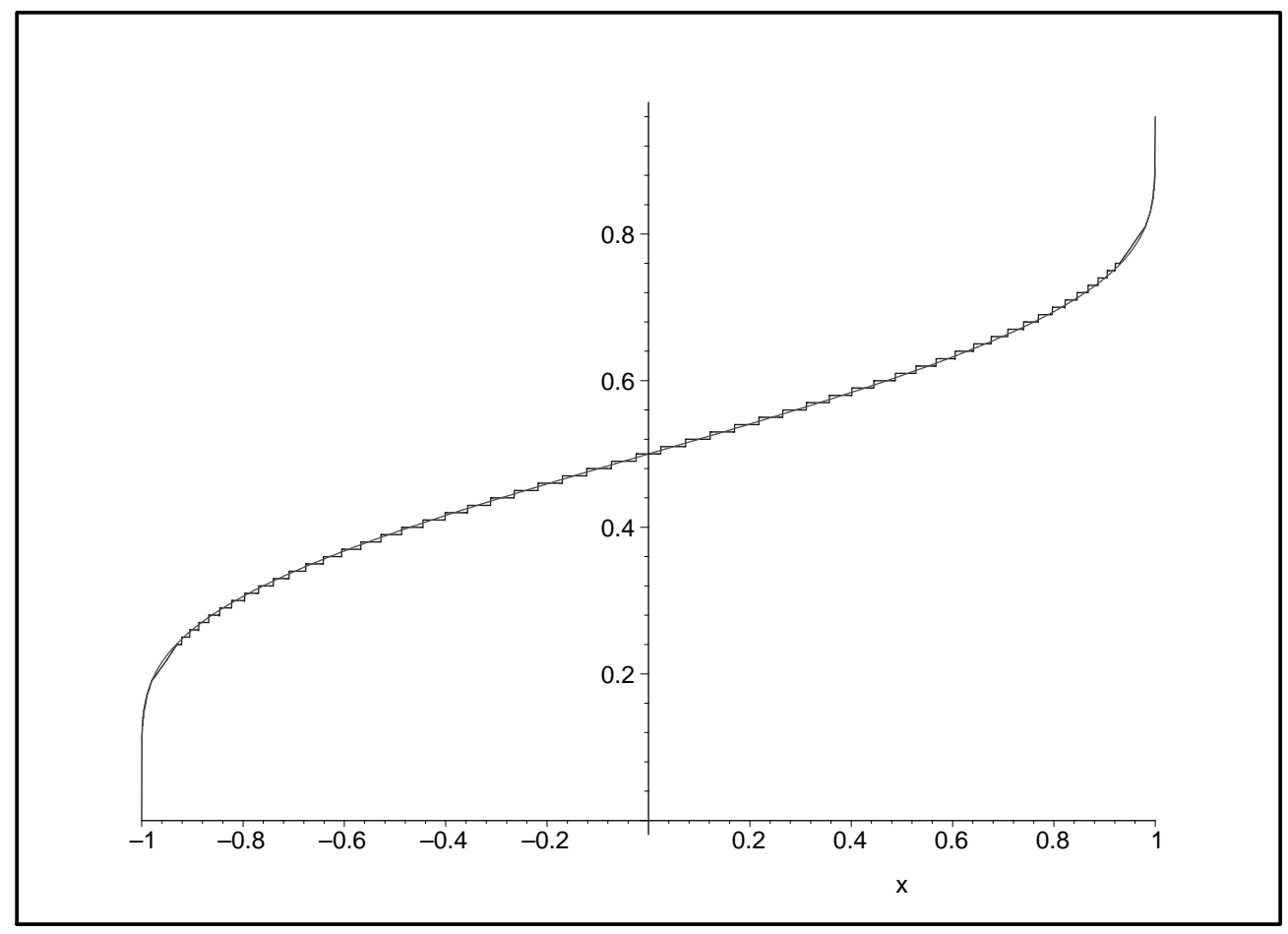

Figure 2: A plot of $\psi_{100}$ (step function) and $\psi$ (solid line) for the tangent polynomials.

Using (68) and $\sigma=0$ in (27) gives $h(t)=1$ and

$$
S(z)=\frac{1}{1-z^{2}}\left[\frac{1}{2} \ln \left(\frac{z-1}{z+1}\right)+C\right]^{-1} .
$$

As $z \rightarrow \infty$, we have

$$
\frac{1}{1-z^{2}}\left[\frac{1}{2} \ln \left(\frac{z-1}{z+1}\right)+C\right]^{-1} \sim-\frac{1}{C z^{2}}-\frac{1}{C^{2} z^{3}},
$$

and hence we need to take $C=0$. Thus,

$$
S(z)=\frac{2}{\left(1-z^{2}\right)}\left[\ln \left(\frac{z-1}{z+1}\right)\right]^{-1} .
$$

From 49, we have

$$
S(z)=\int_{-1}^{1} \frac{d \psi(t)}{z-t}, \quad z \notin[-1,1]
$$

with

$$
\psi^{\prime}(t)=\frac{2}{\left(1-t^{2}\right)}\left[\pi^{2}+\ln ^{2}\left(\frac{1+t}{1-t}\right)\right]^{-1} .
$$


Integrating, we obtain

$$
\psi(t)=\frac{1}{\pi} \arctan \left[\frac{1}{\pi} \ln \left(\frac{1+t}{1-t}\right)\right]+\frac{1}{2}, \quad t \in(-1,1) .
$$

We conclude that

$$
\lim _{n \rightarrow \infty} \frac{1}{n} \#\left\{\text { zeros of } Q_{n}(x) \text { in }[a, b]\right\}=\psi(b)-\psi(a), \quad[a, b] \subset[-1,1] .
$$

In Figure 2, we plot the zero counting distribution $\psi_{100}(t)$ defined in (47) and the distribution function $\psi(t)$ defined in 69 .

Remark 10 To our knowledge, this is the first time that the asymptotic zero distribution (69) has been obtained.

\subsection{Inverse error function polynomials}

Let's consider the polynomials $P_{n}(x)$ defined by $P_{0}(x)=1$ and

$$
P_{n+1}(x)=P_{n}^{\prime}(x)+(n+1) x P_{n}(x) .
$$

The polynomials $P_{n}(x)$ arise in the computation of higher derivatives of the inverse error function [10]. Since they have purely imaginary zeros, we set

$$
P_{n}(x)=n !(\mathrm{i})^{n} Q_{n}(-\mathrm{i} x),
$$

and obtain a family of monic polynomials with real zeros, satisfying

$$
Q_{n+1}(x)=-\frac{1}{n+1} Q_{n}^{\prime}(x)+x Q_{n}(x) .
$$

Using the results in [9], we know that the largest zero of $Q_{n}(x)$ is (roughly) of the order

$$
x_{n, n}=O(\sqrt{2 \ln (n)}), \quad n \rightarrow \infty
$$

See Figure 3, where we compare the values of $x_{n, n}$ with the function $\sqrt{2 \ln (n)}$.

If we take

$$
\phi(n)=\sqrt{2 \ln (n)},
$$

then $\phi(n)$ is slowly varying (at infinity), and we get

$$
\lim _{n \rightarrow \infty} \frac{n}{\phi(n)^{2}} A_{n}(\phi(n) x)=0=a(x), \quad \lim _{n \rightarrow \infty} \frac{1}{\phi(n)} B_{n}(\phi(n) x)=x=b(x) .
$$

We are in the case $\sigma=0$, and hence the differential equation (24) becomes

$$
z S-1=0
$$

so that $S(z)=1 / z$. This is the Stieltjes transform of the degenerate measure with mass one at 0 (the Dirac measure at 0 ) which means that the asymptotic distribution of the scaled zeros $\left\{x_{k, n} / \phi(n), 1 \leq k \leq n\right\}$ is concentrated at the origin. 


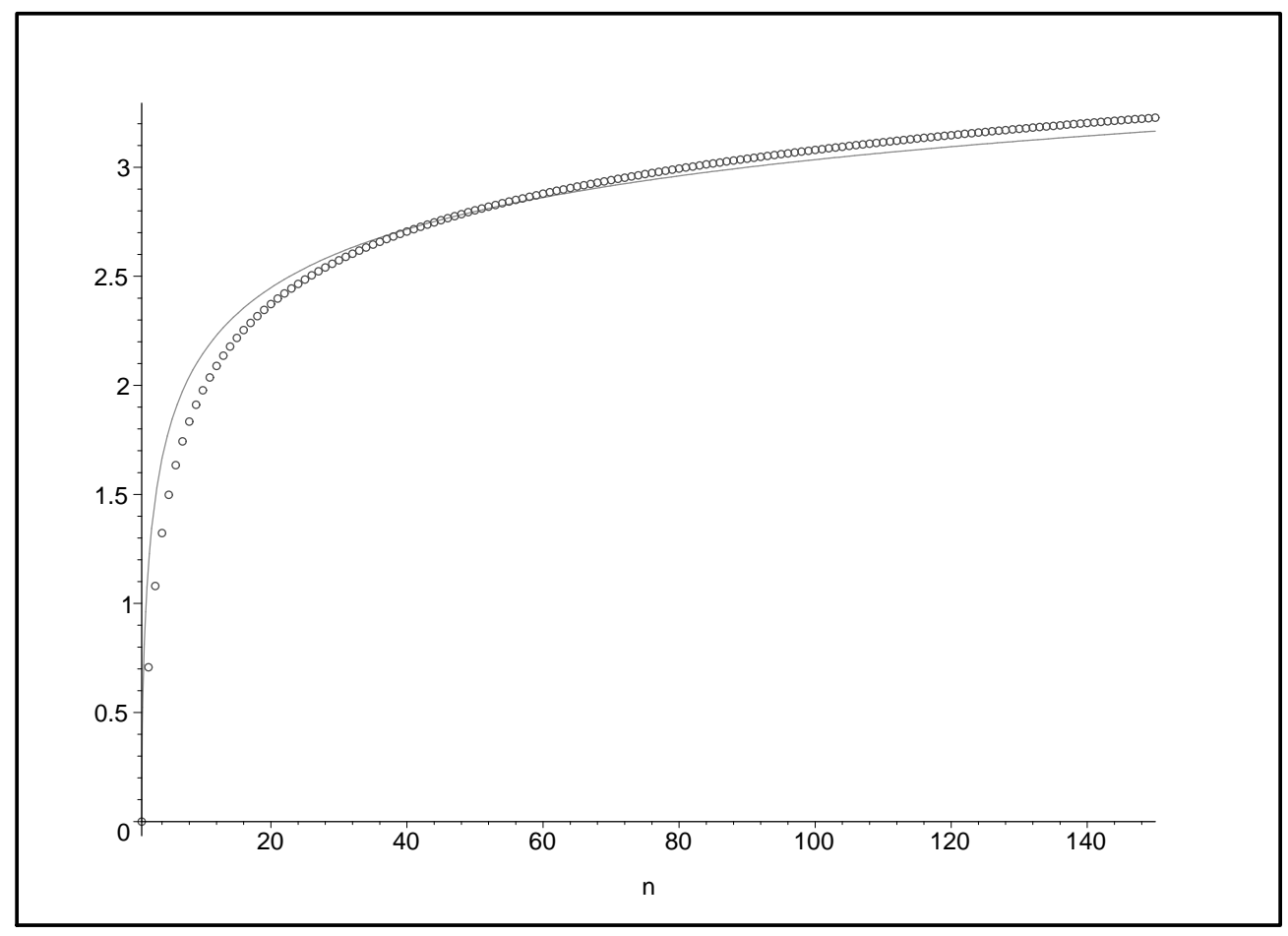

Figure 3: A plot of the largest zero $x_{n, n}$ of the inverse error function polynomials (ooo) and the function $\sqrt{2 \ln (n)}$ (solid line).

If we don't use any scaling, i.e., if we take $\phi(n)=1$, then

$$
\lim _{n \rightarrow \infty} n A_{n}(x)=-1=a(x), \quad \lim _{n \rightarrow \infty} B_{n}(x)=x=b(x),
$$

and again $\sigma=0$, but the zeros of $Q_{n}(x)$ are not bounded and in particular the zeros are not in a compact interval $[a, b]$ for every $1 \leq k \leq n$ and $n \in \mathbb{N}$, so that the conditions of Theorem 2 are not satisfied. However, what we really need is that the sequence of zero counting distributions $\psi_{n}$, defined in (47), has a subsequence that converges weakly to a probability distribution. This will be the case when $\left(\psi_{n}\right)_{n}$ is a tight sequence (Prohorov's theorem, see [2, Thm. 6.1]). Theorems 2 and 3 still hold for unbounded zeros, provided the sequence $\left(\psi_{n}\right)_{n}$ is tight. This is indeed the case for these polynomials.

Definition 11 The sequence of measures $\left(\psi_{n}\right)_{n \geq 1}$ is said to be tight if for all $\varepsilon>0$ there exists a compact set $K_{\varepsilon}$, such that

$$
\psi_{n}\left(K_{\varepsilon}\right)>1-\varepsilon, \quad \forall n \geq 1 .
$$

Proposition 12 The sequence of zero counting distributions corresponding to the Inverse Error Polynomials defined by (70) is tight. 
Proof. Let's write the monic polynomials $Q_{n}(x)$ in the form

$$
Q_{n}(x)=x^{n}+q_{1}(n) x^{n-1}+q_{2}(n) x^{n-2}+\cdots+q_{n}(n) .
$$

Using the results in [6], we have $q_{1}(n)=0$ and

$$
q_{2}(n)=-\sum_{j=0}^{n-1} \frac{j}{j+1}=H_{n}-n,
$$

where $H_{n}$ is the $n$-th harmonic number, defined by

$$
H_{n}=\sum_{j=1}^{n} \frac{1}{j} .
$$

From Newton's identities for the zeros of $Q_{n}(x)$, we know that

$$
\sum_{j=1}^{n}\left(x_{j, n}\right)^{2}=\left(\sum_{j=1}^{n} x_{j, n}\right)^{2}-2 \sum_{j<k} x_{j, n} x_{k, n}=q_{1}^{2}(n)-2 q_{2}(n) .
$$

Let $\varepsilon>0$ and

$$
K_{\varepsilon}=\left[-\sqrt{\frac{2}{\varepsilon}}, \sqrt{\frac{2}{\varepsilon}}\right] .
$$

Using Chebyshev's inequality, we have

$$
\psi_{n}\left(\mathbb{R} \backslash K_{\varepsilon}\right)=\int_{|x|>\sqrt{2 / \varepsilon}} d \psi_{n}(x) \leq \frac{\varepsilon}{2} \int_{R} x^{2} d \psi_{n}(x)=\frac{\varepsilon}{2} \frac{1}{n} \sum_{j=1}^{n}\left(x_{j, n}\right)^{2} .
$$

From 72 and $(73)$ we conclude that

$$
\psi_{n}\left(\mathbb{R} \backslash K_{\varepsilon}\right) \leq \frac{\varepsilon}{2} \frac{2}{n}\left(n-H_{n}\right)<\varepsilon,
$$

since $H_{n}>0$.

Without any scaling, we have (71) and since $\sigma=0$ the differential equation (24) becomes

$$
S^{\prime}(z)=S^{2}(z)-z S(z)+1 .
$$

The required solution is

$$
S(z)=z+\exp \left(\frac{z^{2}}{2}\right)\left[\mathrm{i} \sqrt{\frac{\pi}{2}} \operatorname{erf}\left(\frac{\mathrm{i} z}{\sqrt{2}}\right)-C\right]^{-1},
$$

where $\operatorname{erf}(\cdot)$ is the error function defined by [21, 7.2.1]

$$
\operatorname{erf}(z)=\frac{2}{\sqrt{\pi}} \int_{0}^{z} \exp \left(-t^{2}\right) d t
$$


with asymptotic behavior [21, 7.12.1]

$$
\operatorname{erf}(z) \sim 1-\frac{1}{\sqrt{\pi}} \exp \left(-z^{2}\right)\left(\frac{1}{z}-\frac{1}{2 z^{3}}+\cdots\right), \quad z \rightarrow \infty, \quad|\arg (z)|<\frac{3 \pi}{4}
$$

and

$$
\operatorname{erf}(-z) \sim-1+\frac{1}{\sqrt{\pi}} \exp \left(-z^{2}\right)\left(\frac{1}{z}-\frac{1}{2 z^{3}}+\cdots\right), \quad z \rightarrow \infty, \quad|\arg (z)|<\frac{3 \pi}{4} .
$$

Along the imaginary axis, we thus have

$$
S(\mathrm{i} t)=\mathrm{i} t+\exp \left(-\frac{t^{2}}{2}\right)\left[\mathrm{i} \sqrt{\frac{\pi}{2}} \operatorname{erf}\left(\frac{-t}{\sqrt{2}}\right)-C\right]^{-1},
$$

and

$$
S(-\mathrm{i} t)=-\mathrm{i} t+\exp \left(-\frac{t^{2}}{2}\right)\left[\mathrm{i} \sqrt{\frac{\pi}{2}} \operatorname{erf}\left(\frac{t}{\sqrt{2}}\right)-C\right]^{-1} .
$$

In order to have $z S(z) \rightarrow 1$ as $z \rightarrow \infty$ we conclude that we need to choose $C=-\mathrm{i} \sqrt{\frac{\pi}{2}}$ in the upper half plane and $C=\mathrm{i} \sqrt{\frac{\pi}{2}}$ in the lower half plane. Thus,

$$
S(z)=z-\mathrm{i} \sqrt{\frac{2}{\pi}} \exp \left(\frac{z^{2}}{2}\right)\left[\operatorname{erf}\left(\frac{\mathrm{i} z}{\sqrt{2}}\right) \pm 1\right]^{-1}, \quad z \in \mathbb{C} \backslash \mathbb{R} .
$$

Using the identity [21, 7.5.1]

$$
\operatorname{erf}(\mathrm{i} z)=\frac{2 \mathrm{i}}{\sqrt{\pi}} \exp \left(z^{2}\right) \operatorname{daw}(z)
$$

where daw $(\cdot)$ is Dawson's integral defined by [21, 7.2.5]

$$
\operatorname{daw}(z)=\int_{0}^{z} \exp \left(t^{2}-z^{2}\right) d t
$$

we can write

$$
S(z)= \begin{cases}z-\sqrt{\frac{2}{\pi}} \exp \left(\frac{z^{2}}{2}\right) \frac{\frac{2}{\sqrt{\pi}} \exp \left(\frac{z^{2}}{2}\right) \operatorname{daw}\left(\frac{z}{\sqrt{2}}\right)+\mathrm{i}}{\frac{4}{\pi} \exp \left(z^{2}\right) \operatorname{daw}^{2}\left(\frac{z}{\sqrt{2}}\right)+1}, \quad \operatorname{Im}(z)>0, \\ z-\sqrt{\frac{2}{\pi}} \exp \left(\frac{z^{2}}{2}\right) \frac{\frac{2}{\sqrt{\pi}} \exp \left(\frac{z^{2}}{2}\right) \operatorname{daw}\left(\frac{z}{\sqrt{2}}\right)-\mathrm{i}}{\frac{4}{\pi} \exp \left(z^{2}\right) \operatorname{daw}^{2}\left(\frac{z}{\sqrt{2}}\right)+1}, \quad \operatorname{Im}(z)<0 .\end{cases}
$$

From 49), we have

$$
S(z)=\int_{-\infty}^{\infty} \frac{d \psi(t)}{z-t}, \quad z \notin \mathbb{R}
$$

with

$$
\psi^{\prime}(t)=\sqrt{\frac{2}{\pi}} \exp \left(\frac{t^{2}}{2}\right)\left[4 \exp \left(t^{2}\right) \operatorname{daw}^{2}\left(\frac{t}{\sqrt{2}}\right)+\pi\right]^{-1}
$$




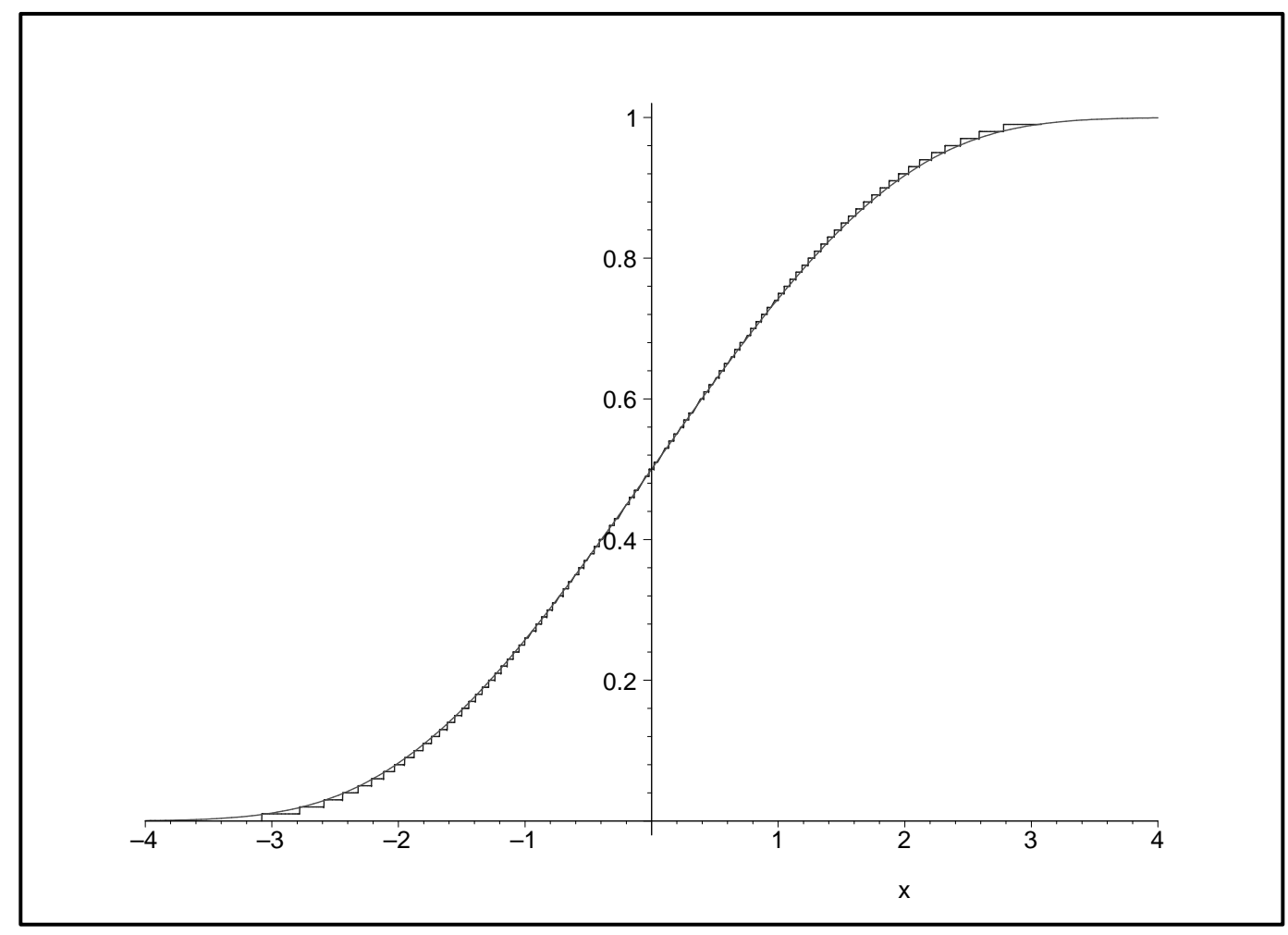

Figure 4: A plot of $\psi_{100}$ (step function) and $\psi$ (solid line) for the inverse error function polynomials.

But since

$$
\frac{d}{d t} \exp \left(\frac{t^{2}}{2}\right) \text { daw }\left(\frac{t}{\sqrt{2}}\right)=\frac{1}{\sqrt{2}} \exp \left(\frac{t^{2}}{2}\right)
$$

we obtain

$$
\psi(t)=\frac{1}{\pi} \arctan \left[\frac{2}{\sqrt{\pi}} \exp \left(\frac{t^{2}}{2}\right) \text { daw }\left(\frac{t}{\sqrt{2}}\right)\right]+\frac{1}{2}, \quad t \in \mathbb{R} .
$$

We conclude that

$$
\lim _{n \rightarrow \infty} \frac{1}{n} \#\left\{\text { zeros of } Q_{n}(x) \text { in }[a, b]\right\}=\psi(b)-\psi(a) .
$$

In Figure 4, we plot the zero counting distributions $\psi_{100}(t)$ defined in (47) and the distribution function $\psi(t)$ defined in $(75)$.

In [9], we analyzed the polynomials $P_{n}(x)$ asymptotically and, among others, we considered the limit $n \rightarrow \infty$, with $x=y / n$ and $y=O(1)$. We obtained the asymptotic 
approximation

$$
P_{n}(x) \sim n^{n} e^{-n} \sqrt{\frac{2 n}{\ln (n)}}\left(\sqrt{\frac{2}{\pi}}\right)^{n}\left[\exp \left(\sqrt{\frac{2}{\pi}} y\right)+(-1)^{n} \exp \left(-\sqrt{\frac{2}{\pi}} y\right)\right],
$$

and therefore

$$
Q_{n}(x) \sim \mathrm{i}^{n} \frac{1}{\sqrt{\pi \ln (n)}}\left(\sqrt{\frac{2}{\pi}}\right)^{n}\left[\exp \left(-\sqrt{\frac{2}{\pi}} n x \mathrm{i}\right)+(-1)^{n} \exp \left(\sqrt{\frac{2}{\pi}} n x \mathrm{i}\right)\right],
$$

where we have used Stirling's formula [21, 5.11.7]

$$
n ! \sim \sqrt{2 \pi n} n^{n} e^{-n}, \quad n \rightarrow \infty
$$

Thus, we get

$$
Q_{n}(x) \sim\left\{\begin{array}{cc}
2\left(-\frac{2}{\pi}\right)^{\frac{n}{2}}[\pi \ln (n)]^{-\frac{1}{2}} \cos \left(\sqrt{\frac{2}{\pi}} n x\right), \quad n \text { is even } \\
-\sqrt{2}\left(-\frac{2}{\pi}\right)^{\frac{n+1}{2}}[\pi \ln (n)]^{-\frac{1}{2}} \sin \left(\sqrt{\frac{2}{\pi}} n x\right), \quad n \text { is odd }
\end{array} .\right.
$$

It follows that $x_{k, n}$ (the zeros of $Q_{n}$ ), are approximated asymptotically by

$$
x_{k, n} \sim\left\{\begin{array}{c}
\frac{\pi^{\frac{3}{2}}}{\sqrt{2}} \frac{k}{n}+\left(\frac{\pi}{2}\right)^{\frac{3}{2}} \frac{1}{n}, \quad n \text { is even } \\
\frac{\pi^{\frac{3}{2}}}{\sqrt{2}} \frac{k}{n}, \quad n \text { is odd }
\end{array}\right.
$$

with $k=0, \pm 1, \pm 2, \ldots$ Hence, the zero counting distribution (47) can be approximated by

$$
d \psi_{n}(t) \sim \frac{\sqrt{2}}{\pi^{\frac{3}{2}}} d t, \quad n \rightarrow \infty
$$

But from 75 , we have

$$
\psi(t) \sim \frac{1}{2}+\frac{\sqrt{2}}{\pi^{\frac{3}{2}}} t, \quad t \rightarrow 0
$$

in agreement with $(76)$.

Remark 13 In [9] we obtained some asymptotic results on the polynomials $P_{n}(x)$, but this is the first time that the asymptotic zero distribution (75) has been considered.

\section{Conclusion}

In this paper we have investigated the asymptotic zero distribution of a family of polynomials satisfying a differential-difference equation of the form

$$
P_{n+1}(x)=A_{n}(x) P_{n}^{\prime}(x)+B_{n}(x) P_{n}(x), \quad n \geq 0,
$$

where $A_{n}$ are polynomials of degree at most 2 and $B_{n}$ are polynomials of degree at most 1. We have shown that, assuming the zeros of the polynomials interlace and after appropriate scaling using some regularly varying function $\phi(n)$, the Stieltjes transform of 
the asymptotic zero distribution satisfies a differential equation of Riccati or Abel type, which can be solved explicitly. We have illustrated this result for the classical orthogonal polynomials of Jacobi, Laguerre, and Hermite, for which the asymptotic zero distribution is already well known, but also for three families of polynomials which are not orthogonal polynomials: the Bell polynomials, the tangent polynomials, and polynomials related to the inverse error function. One of the main ingredients in this paper is Theorem 2 which shows that the sequence of ratios $\frac{\phi(n)}{n} \frac{P_{[n t]}^{\prime}(\phi(n) x)}{P_{[n t]}(\phi(n) x)}$ with regularly varying scaling $\phi(n)$ is relatively compact in the Skorohod metric on $D[0,1]$.

\section{Acknowledgments}

The authors are very grateful to the referees for their careful reading of the paper and their constructive remarks.

\section{References}

[1] E. T. Bell. Exponential polynomials. Ann. of Math. (2), 35(2):258-277, 1934.

[2] P. Billingsley. Convergence of probability measures. John Wiley \& Sons Inc., New York, 1968.

[3] K. N. Boyadzhiev. Derivative polynomials for tanh, tan, sech and sec in explicit form. Fibonacci Quart., 45(4):291-303, 2008.

[4] R. M. Corless, G. H. Gonnet, D. E. G. Hare, D. J. Jeffrey, and D. E. Knuth. On the Lambert $W$ function. Adv. Comput. Math., 5(4):329-359, 1996.

[5] R. M. Corless, D. J. Jeffrey, and D. E. Knuth. A sequence of series for the Lambert $W$ function. In Proceedings of the 1997 International Symposium on Symbolic and Algebraic Computation (Kihei, HI), pages 197-204 (electronic), New York, 1997. ACM.

[6] D. Dominici. Some properties of the inverse error function. In Tapas in experimental mathematics, volume 457 of Contemp. Math., pages 191-203. Amer. Math. Soc., Providence, RI, 2008.

[7] D. Dominici. Asymptotic analysis of the Bell polynomials by the ray method. J. Comput. Appl. Math., doi:10.1016/j.cam.2009.02.082.

[8] D. Dominici, K. Driver, and K. Jordaan. Polynomial solutions of differentialdifference equations. J. Approx. Theory, 163(1):41-48, 2011.

[9] D. Dominici and C. Knessl. Asymptotic analysis of a family of polynomials associated with the inverse error function. Rocky Mountain J. Math., 42(3):847-872, 2012.

[10] D. E. Dominici. The inverse of the cumulative standard normal probability function. Integral Transforms Spec. Funct., 14(4):281-292, 2003. 
[11] F. Dubeau and J. Savoie. More on the roots of Euler-Frobenius polynomials. Ann. Sci. Math. Québec, 22(1):31-42, 1998.

[12] C. Elbert. Strong asymptotics of the generating polynomials of the Stirling numbers of the second kind. J. Approx. Theory, 109(2):198-217, 2001.

[13] C. Elbert. Weak asymptotics for the generating polynomials of the Stirling numbers of the second kind. J. Approx. Theory, 109(2):218-228, 2001.

[14] P. Henrici. Applied and computational complex analysis. Vol. 2. Wiley Interscience [John Wiley \& Sons], New York, 1977.

[15] G. Hetyei. Tchebyshev triangulations of stable simplicial complexes. J. Combin. Theory Ser. A, 115(4):569-592, 2008.

[16] M. E. Hoffman. Derivative polynomials for tangent and secant. Amer. Math. Monthly, 102(1):23-30, 1995.

[17] G. A. Kalugin, D. J. Jeffrey, R. M. Corless, and P. B. Borwein. Stieltjes and other integral representations for functions of Lambert $W$. Integral Transforms Spec. Funct., 23(8):581-593, 2012.

[18] E. Kamke. Differentialgleichungen. Lösungsmethoden und Lösungen. Band I. Gewöhnliche Differentialgleichungen. Mathematik und ihre Anwendungen in Physik und Technik. Band 18. Akademische Verlagsgesellschaft, Leipzig, 1944. 3d ed.

[19] R. Koekoek, P. A. Lesky, and R. F. Swarttouw. Hypergeometric orthogonal polynomials and their q-analogues. Springer Monographs in Mathematics. Springer-Verlag, Berlin, 2010.

[20] W. Lang. On sums of powers of zeros of polynomials. J. Comput. Appl. Math. 89 (1998), 237-256.

[21] F. W. J. Olver, D. W. Lozier, R. F. Boisvert, and C. W. Clark, editors. NIST handbook of mathematical functions. U.S. Department of Commerce National Institute of Standards and Technology, Washington, DC, 2010.

[22] A. D. Polyanin and V. F. Zaitsev. Handbook of exact solutions for ordinary differential equations. Chapman \& Hall/CRC, Boca Raton, FL, second edition, 2003.

[23] W. T. Reid. Riccati differential equations. Academic Press, New York, 1972.

[24] I. J. Schoenberg. On the zeros of the generating functions of multiply positive sequences and functions. Ann. of Math. (2), 62:447-471, 1955.

[25] R. P. Stanley. Log-concave and unimodal sequences in algebra, combinatorics, and geometry. In Graph theory and its applications: East and West (Jinan, 1986), volume 576 of Ann. New York Acad. Sci., pages 500-535. New York Acad. Sci., New York, 1989.

[26] G. Szegő. Orthogonal polynomials. American Mathematical Society, Providence, R.I., fourth edition, 1975. 
[27] W. Van Assche. Asymptotics for orthogonal polynomials, volume 1265 of Lecture Notes in Mathematics. Springer-Verlag, Berlin, 1987.

[28] H. S. Wall. Analytic Theory of Continued Fractions. D. Van Nostrand Company, Inc., New York, N. Y., 1948.

[29] H. S. Wilf. Generatingfunctionology. A K Peters Ltd., Wellesley, MA, third edition, 2006. 